\title{
Short-Term Results of Hybrid Closed-Wedge High Tibial Osteotomy: A Case Series with a Minimum 3-Year Follow-up
}

\author{
Hidetomo Saito, $\mathrm{MD}, \mathrm{PhD}^{1,2}$, Kimio Saito, $\mathrm{MD}, \mathrm{PhD}^{1,2}$, Yoichi Shimada, $\mathrm{MD}, \mathrm{PhD}^{1}$, Toshiaki Yamamura, $\mathrm{MD}^{3}$, \\ Shin Yamada, $\mathrm{MD}, \mathrm{PhD}^{1}$, Takahiro Sato, $\mathrm{MD}^{3}$, Koji Nozaka, $\mathrm{MD}, \mathrm{PhD}^{1}$, Hiroaki Kijima, $\mathrm{MD}, \mathrm{PhD}^{1,2}$, and \\ Naohisa Miyakoshi, MD, $\mathrm{PhD}^{1}$ \\ ${ }^{1}$ Department of Orthopedic Surgery, Akita University Graduate School of Medicine, Akita; ${ }^{2}$ Akita Sports Arthroscopy and Knee Group, Akita; ${ }^{3}$ Sapporo Sports Clinic, \\ Sapporo, Japan
}

\begin{abstract}
Purpose: High tibial valgus osteotomy (HTO) is a well-established surgical procedure for patients with medial compartment osteoarthritis (OA) of the knee. The hybrid closed-wedge HTO (CWHTO) procedure permits extensive correction in patients with severe deformities or patellofemoral joint OA. The aim of this study was to report the short-term results in a consecutive series of patients treated with hybrid CWHTO.

Materials and Methods: We retrospectively evaluated the clinical outcomes and radiographic parameters in 29 consecutive knees that underwent hybrid CWTHO to correct medial compartment OA at an average follow-up of 52.6 months. Clinical outcomes were assessed using the Lysholm score and knee scoring system of the Japanese Orthopedic Association (JOA). The Kellgren-Lawrence grading system and pre- and postoperative mechanical axis (MA), femorotibial angle (FTA), posterior tibial slope, and patella height were assessed.

Results: The FTA and MA significantly changed from $180.7^{\circ}$ to $170.4^{\circ}$ and from $22.0^{\circ}$ to $60.2^{\circ}$, respectively. No significant differences were observed between the mean pre- and postoperative posterior tibial slope, Insall-Salvati ratio, or Caton-Deschamps index. The postoperative JOA and Lysholm scores significantly improved from 76.7 to 95.8 and from 58.8 to 90.2 , respectively.

Conclusions: Satisfactory outcomes can be achieved with hybrid CWHTO in patients with medial OA.
\end{abstract}

Keywords: Knee, Osteoarthritis, Osteotomy, Closed-wedge, Hybrid

\section{Introduction}

High tibial valgus osteotomy (HTO) is a well-established surgical procedure for patients with medial compartment osteoarthritis $(\mathrm{OA})$ of the knee ${ }^{1-3)}$. Several types of HTO have been reported including medial open-wedge HTO (OWHTO) $)^{4,5)}$ and lateral

Received January 16, 2018; Revised April 16, 2018;

Accepted April 24, 2018

Correspondence to: Hidetomo Saito, $\mathrm{MD}, \mathrm{PhD}$

Department of Orthopedic Surgery, Akita University Graduate School of Medicine, Hondo 1-1-1, Akita 010-8543, Japan

Tel: +81-18-884-6148, Fax: +81-18-884-2617

E-mail: hidetomo@doc.med.akita-u.ac.jp

This is an Open Access article distributed under the terms of the Creative Commons Attribution Non-Commercial License (http://creativecommons.org/licenses/by-nc/4.0/) which permits unrestricted non-commercial use, distribution, and reproduction in any medium, provided the original work is properly cited. closed-wedge HTO (CWHTO $)^{6}$. Currently, OWHTO, which is a simpler procedure, is widely used to correct varus deformities in patients with early- or middle-stage knee $\mathrm{OA}^{3,7-9)}$. Takeuchi et al. $^{10)}$ have now developed a hybrid CWHTO procedure and reported on the postoperative rehabilitation outcomes of this novel technique.

The hybrid CWHTO method is based on the conventional CWHTO approach and has several advantages over traditional CWHTO and OWHTO ${ }^{10)}$. The terms "hybrid" in this context denotes a combination of the lateral closed-wedge and medial open-wedge procedures created by a unique hinge point. First, the unique hinge point facilitates less wedged bone loss at the lateral side and greater correction of the osteotomy. This lower bone loss could be beneficial in terms of reduced leg shortening. In addition, step-off is avoided by using an oblique osteotomy, permitting a firm contact with the lateral and posterior cortex of the osteotomy site, as well as providing biomechanical advantages. 
Second, the biplanar osteotomy, first described by Staubli et al. ${ }^{5)}$ and colleagues enables a larger bone contact area to be obtained, which is beneficial for the primary stability of the bone-implant structure and prevention of malrotational correction ${ }^{11}$. Moreover, the use of a rigid locking compression plate internal fixation device increases the stability of the osteotomy site and allows early full weight bearing walking after surgery ${ }^{12)}$.

Accordingly, the hybrid CWHTO method enables more extensive correction in patients with severe deformities compared with OWHTO and can be used in cases of a flexion contracture of more than $15^{\circ}$, which is a contraindication for OWHTO. Additionally, CWHTO produces less lowering of the patella and lower pressure increases at the patellofemoral joint compared with OWHTO ${ }^{13,14}$. To our knowledge, however, there have been no reports on the outcomes of hybrid CWHTO other than by the original authors ${ }^{10)}$. In our present study, we have for the first time investigated the short-term results of this procedure in a consecutive series of patients.

\section{Materials and Methods}

\section{General Considerations}

We retrospectively evaluated pain, function, and alignment in a single group of 29 consecutive knees from 26 patients (15 females and 11 males) that underwent hybrid CWTHO for medial compartment OA of the knee between 2012 and 2013. Among these 29 subject knees, 2 knees underwent simultaneous anterior cruciate reconstruction using a bone-patella tendon-bone (BTB) graft $^{15)}$. The demographic data for these patients are presented in Table 1. We only evaluated knees that had been operated on by the same surgeon (HS) to avoid possible learning curve effects. The cartilage status was arthroscopically evaluated in all knees prior to the osteotomy using the Outerbridge classification ${ }^{16)}$. In three patients, simultaneous hybrid CWHTO was selected to treat extensive bilateral deformities. Finally, all patients who could be followed-up for more than 6 months were included in our current analysis. The average patient age was 56.7 years (range, 39 to 77 years). The mean follow-up period was 52.6 months (range, 41.9 to 68.1 months) with a follow-up rate of $100 \%$ (29/29 knees).

\section{Preoperative Planning}

\section{1) Step 1}

We first obtained a radiograph of the anteroposterior view of the whole leg in the standing position. The corrected X-ray is the scaffold for further planning. Hybrid CWHTO is then performed
Table 1. Characteristics of Patients

\begin{tabular}{lc}
\hline \multicolumn{1}{c}{ Characteristic } & Value \\
\hline No. of patients $(\%)$ & $15(57.7)$ \\
Male & $11(42.3)$ \\
Female & $56.7(39-77)$ \\
Age $(\mathrm{yr})$, mean (range) & $70.2 \pm 15.0$ \\
Weight $(\mathrm{kg})$, mean \pm SD & $162.5 \pm 7.6$ \\
Height $(\mathrm{cm})$, mean \pm SD & $31.7 \pm 6.1$ \\
BMI $\left(\mathrm{kg} / \mathrm{cm}^{2}\right)$, mean \pm SD &
\end{tabular}

SD: standard deviation, BMI: body mass index.

using this radiographic image of the leg where the postoperative weight bearing line (WBL) passes through $62 \%$ laterally from the medial edge of the tibial plateau. The preoperative WBL is detected by drawing a line from the center of the femoral head to the middle point of the proximal talar joint surface (broken line) (Fig. 1A).

2) Step 2

The correction angle of the osteotomy and the height of the osteotomy gap are defined using the planning technique described by Miniaci et al. ${ }^{177}$. The new WBL is drawn from the center of the femoral head, passing the knee at the point defined in step 1 to the height of the ankle joint line (Fig. 1B). The hinge point of the osteotomy $(\mathrm{H})$ is defined with respect to the length of the first cut line (100\%) at the proximal tibia and is set at about $33 \%$ (1/3) from the medial border of the first cut line of the tibia on the proximal osteotomy line and connected distally with the new and the old center of the ankle joint. The correction angle can be measured between the two lines A and B as shown in Fig. 1C. The angle of correction at the proximal tibia corresponds to angle a between lines A and B (Fig. 1D).

\section{Surgical Indication}

In our present case series, we performed hybrid CWHTO to treat all patients with medial OA regardless of the degree of $\mathrm{OA}$ or extent of the deformity.

\section{Surgical Technique}

The aim of the hybrid CWTHO approach is to achieve a mechanical axis (MA) from the center of the hip to the center of the ankle through $62 \%$ medial to the tibial plateau and a femorotibial angle (FTA) between the anatomical axes of the femur and tibia of $170^{\circ}$. The correction angles were determined in accordance with the Miniaci method ${ }^{17}$. The hybrid CWHTO procedure, 
with preservation of the proximal tibiofibular joint, was performed using a special fibular osteotomy procedure that we developed. This was done at the middle of the fibula after release and preparation of the extensor muscles of the leg with avoidance of the lateral peroneal nerve. The fibula was placed to the left after making some slots of 10-20 $\mathrm{mm}$ in the lateral aspect of the fibula using a chisel or bone saw. These slots were then gently extended proximally and distally under the periosteum with a 10 $\mathrm{mm}$ chisel to $50-60 \mathrm{~mm}$ in length so that they could subsequently be incurved and bent towards the hybrid CWHTO (Fig. 2). The first tibial oblique cut then ran from the lateral to the medial cortex, which is approximately $35 \mathrm{~mm}$ distal to the lateral proxi-
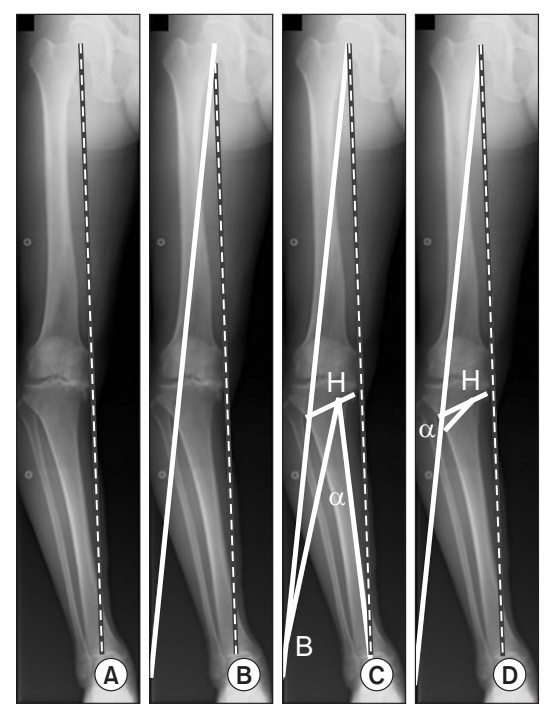

Fig. 1. Preoperative planning of hybrid closed-wedge high tibial valgus osteotomy. (A) The weight bearing line passes medially from medial cortex of tibial plateau (dashed line). (B) A new weight bearing line (solid line) that passes through both the point $62 \%$ from medial cortex in tibial plateau and the center of femoral head was drawn. (C) The hinge point $(\mathrm{H})$ was defined, and the correction angle $(\alpha)$ was measured. (D) Finally, the amount of bone wedge resection was determined. mal tibial joint surface. The medial target position was about 15 $\mathrm{mm}$ distal to the medial proximal tibial joint surface as well as the deep layer attachment site of the distal portion of the medial collateral ligament (MCL; the first oblique line was drawn from the lateral cortex to the medial target position). Two Kirschner wires were then inserted along the line of the first cut under fluoroscopy.

After the hinge point, where the proximal tibial osteotomy line divided by 2 to 1 was formed, the distal osteotomy line (the second osteotomy line) was determined using a special goniometer (KONNO Corporation, Akita, Japan) (Fig. 3), and two Kirschner
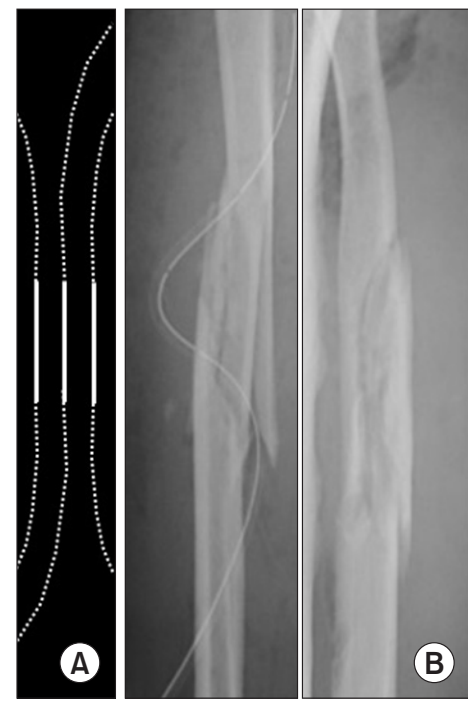

Fig. 2. A new fibular osteotomy. (A) A schematic drawing of incurvated flexiated fibular osteotomy (IFFO). First, three slots were made by a bone saw (solid line). Then, The fibula was comminuted at a length of approximately $50-60 \mathrm{~mm}$ using chisels to preserve the anterior, posterior, and medial periosteum according to the dashed line. (B) Postoperative radiographs of the new fibular osteotomy. This technique enables the fibula to be corrected following tibial osteotomy and a high union rate can be expected using this specialized procedure. We named this method as IFFO.
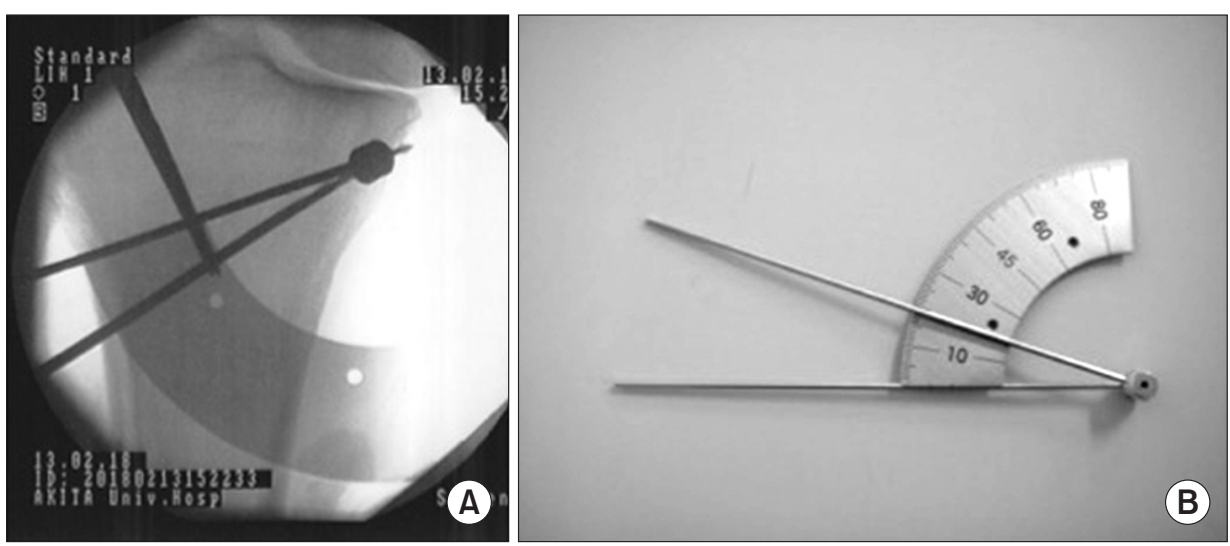

Fig. 3. How to confirm the correction angle during surgery. (A) Correction angle confirmed with a special goniometer under fluoroscopy. (B) A special goniometer (KONNO Corporation) was used to determine the osteotomy line during preoperative planning. 
wires were inserted along this second osteotomy line (Fig. 4A). The wedge between the Kirschner wires was then removed using a bone saw and chisel. The separate ascending cut of the biplanar osteotomy was subsequently performed behind the patellar tendon insertion in the frontal plane, retaining the thickness of the tibial tuberosity at $5 \mathrm{~mm}$ to $10 \mathrm{~mm}$. Finally, the osteotomy was completed with the medial cortex cut according to the first osteotomy line (Fig. 4B). The term "hybrid" was derived from an Xray of the lateral closed-wedge and medial open-wedge style (Fig. 5). With correct preoperative planning, this approach can correct even varus deformities of $185^{\circ}$ or more. The osteotomy fixation was performed with a proximal-lateral tibial plate with five distal screw holes (DePuy Synthes, West Chester, PA, USA), which was developed for proximal tibial fracture repair and always requires prebending by as much as the planned corrective angle. Pre- and postoperative long leg standing X-rays are shown in Fig. 6.

\section{Postoperative Rehabilitation}

From the first postoperative day, all patients begin active and passive range of motion and muscle strengthening exercises after the drainage tube was removed. Range of motion exercises with continuous passive motion were allowed as soon as possible on the same day. One week after surgery, patients were permitted full weight bearing with support equipment, if tolerated.
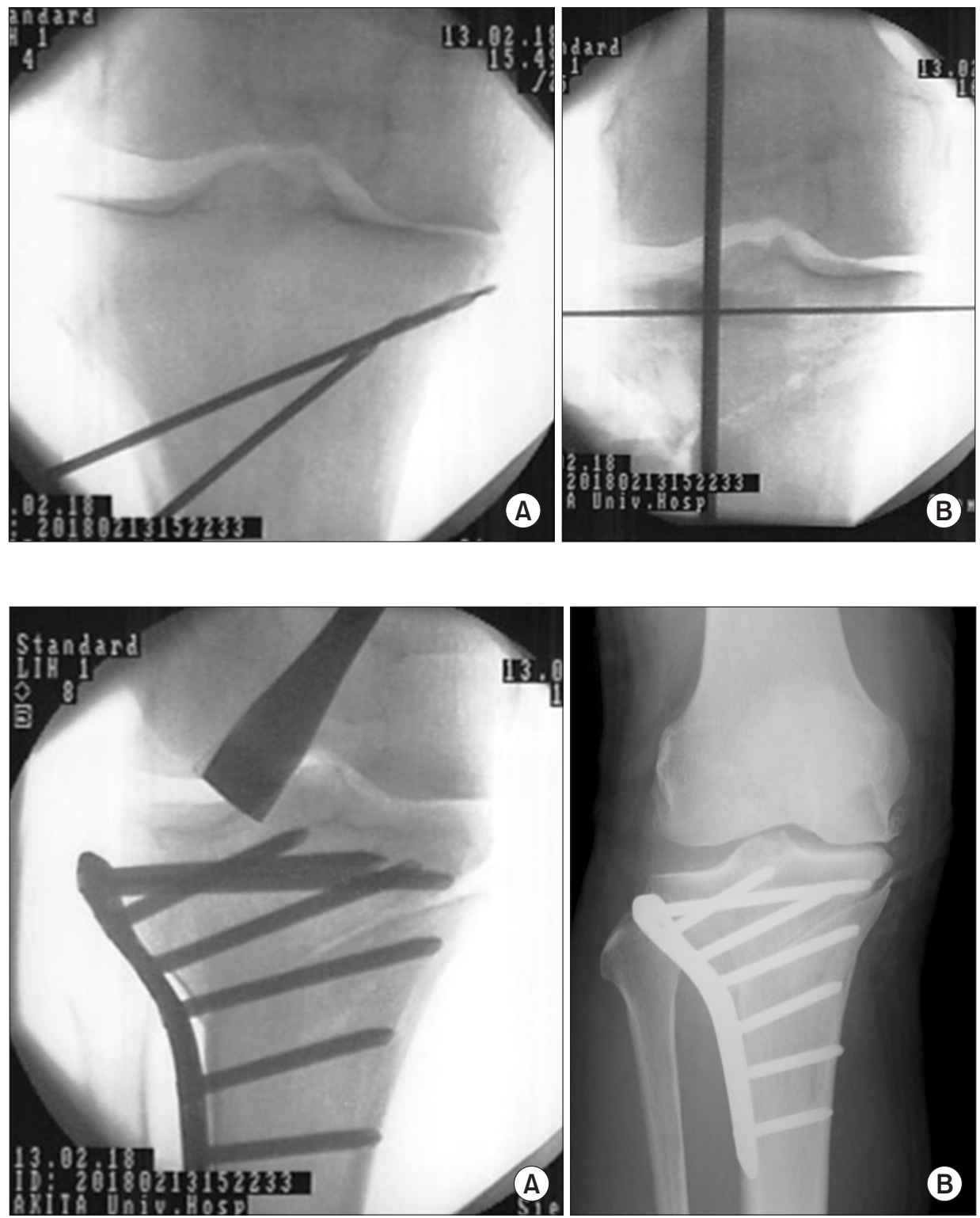

Fig. 4. Intraoperative fluoroscopy. (A) Tip of Kirschner wires crossed at the hinge point. The lateral bone wedge surrounded by Kirschner wires were removed using a bone saw and chisels for correction. (B) After the wedge was removed, manual correction was possible and the hybrid osteotomy (medial open and lateral closed) was completed with respect to the hinge point. The weight bearing line was shifted laterally, passing through around $62 \%$ from medial cortex.

Fig. 5. "Hybrid" (medial open and lateral closed) was completed. (A) Intraoperative fluoroscopy right after locking plate fixation. (B) Postoperative radiograph. 


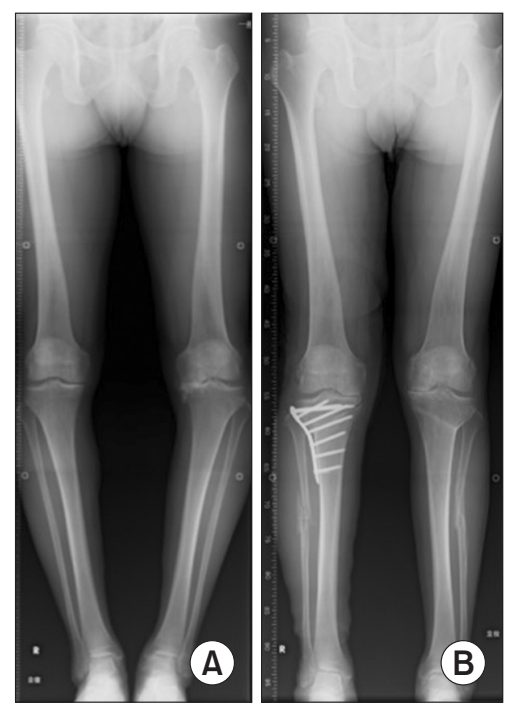

Fig. 6. Pre- and postoperative long leg standing radiographs. (A) The femorotibial angle (FTA) was $184^{\circ}$ on the right side and $191^{\circ}$ on the left side. (B) The FTA was $170^{\circ}$ on the right side and $168^{\circ}$ on the left side after correction.

\section{Radiographic Measurements}

Preoperative and postoperative radiographs included a full leg bilateral weight bearing radiograph, standard Rosenberg view in the standing position, and a gravity sag view ${ }^{18)}$. The preoperative degree of OA was classified according to Kellgren-Lawrence grading. Pre- and postoperative MA and FTA measured on full leg standing X-rays, as well as the posterior tibial slope (PTS), which is the inclination angle of the tibial plateau to the axis of the tibia, the Insall-Salvati ratio, and the Caton-Deschamps index were assessed as indices of patellar height in the gravity sag view. The preoperative and final follow-up radiological grades of OA in patellofemoral compartments were assessed by standing skyline view radiography in accordance with a modified OA grading system $^{19,20)}$.

\section{Follow-up Evaluation}

The Lysholm score ${ }^{21)}$ and knee scoring system of the Japanese Orthopedic Association (JOA ${ }^{22)}$ were investigated at the final follow-up, as well as radiographic measurements such as the FTA, MA, PTS, Insall-Salvati ratio, Caton-Deschamps index, and patellofemoral OA grade. Complications and additional surgery, such as removal of implants, were noted.

\section{Statistical Analysis}

Differences between related measurements were evaluated using a paired Student $t$-test. Difference between preoperative and final follow-up radiological grades of OA in patellofemoral com-
Table 2. Radiographic Osteoarthritis Grade \& Intraoperative Arthroscopic Osteoarthritis Grade

\begin{tabular}{|c|c|c|c|c|}
\hline \multirow{2}{*}{$\begin{array}{c}\text { Kellgren- } \\
\text { Lawrence } \\
\text { grade }\end{array}$} & \multicolumn{2}{|c|}{ Patellofemoral joint } & \multirow{2}{*}{$\begin{array}{c}\text { Femorotibial } \\
\text { joint }\end{array}$} & \multirow{2}{*}{$\begin{array}{l}\text { Outerbridge } \\
\text { classification }\end{array}$} \\
\hline & Preoperative & Postoperative & & \\
\hline Grade 0 & - & - & - & $0(0)$ \\
\hline Grade I & $24(82.8)$ & $22(75.9)$ & $2(6.9)$ & $0(0)$ \\
\hline Grade II & $5(17.2)$ & $7(24.1)$ & $12(41.4)$ & $1(3.4)$ \\
\hline Grade III & $0(0)$ & $0(0)$ & $7(24.1)$ & $15(51.7)$ \\
\hline Grade IV & N/A & N/A & $8(27.6)$ & $13(44.8)$ \\
\hline $\mathrm{p}$-value $\left(\chi^{2}\right)$ & \multicolumn{2}{|c|}{$<0.001(19.0)$} & & \\
\hline
\end{tabular}

Values are presented as number (\%).

Grade 0: normal cartilage; Grade I: cartilage with softening and swelling, Grade II: a partial-thickness defect with fissures on the surface that does not reach subchondral bone or exceeds $1.5 \mathrm{~cm}$ in diameter, Grade III: fissuring to the level of the subchondral bone in an area with a diameter more than $1.5 \mathrm{~cm}$, Grade IV: exposed subchondral bone, N/A: not applicable.

partments was evaluated using Pearson chi-square test. SPSS ver. 17.0 (SPSS Inc., Chicago, IL, USA) was used for statistical analyses, and a p-value of 0.05 or less was considered significant.

\section{Ethical Standards}

All patients provided informed consent prior to their inclusion in the study. The study was approved by the Ethics Committee of our institution (no. 1251) and was performed in accordance with ethical standards.

\section{Results}

The preoperative Kellgren-Lawrence grade of the 29 subject knees and intraoperative Outerbridge classification of the medial compartments are presented in Table 1. The pre- and postoperative FTA significantly changed from $180.7^{\circ}$ (range, $175.0^{\circ}$ to $195.0^{\circ}$ ) to $170.4^{\circ}$ (range, $163.0^{\circ}$ to $\left.177.0^{\circ}\right)(\mathrm{p}<0.001$ ). The mean preoperative MA was $22.0^{\circ}$ medial to the tibial plateau (range, $-15.3^{\circ}$ to $39.5^{\circ}$ ) and the mean postoperative MA was $60.2^{\circ}$ (range, $37.3^{\circ}$ to $\left.80.3^{\circ}\right)(\mathrm{p}<0.001)$. No significant differences were observed between the mean pre- and postoperative PTS, InsallSalvati ratio or Caton-Deschamps index (Table 1). From a comparison of the preoperative and final follow-up radiological OA grades in the patellofemoral compartments, a significant deterioration of the OA grade was observed in two of the 29 knees (Table 2). Simultaneous hybrid CWHTO and ACL reconstruction with BTB graft was conducted in these 2 knees, but they did not reveal any patellofemoral symptoms. The OA grade of the remaining 27 


\section{Saito et al. Short-Term Results of Hybrid Closed-Wedge High Tibial Osteotomy}

knees was unchanged.

No patellar subluxation was observed on the pre- and postoperative X-rays in our current study series. The JOA and Lysholm scores significantly improved from 76.7 to 95.8 and from 58.8 to 90.2, respectively (Table 3). All of the fibular osteotomies among our subject knees were successfully united and no knee required additional surgery due to delayed union or nonunion. This new fibular osteotomy method, which exhibits a high bone union rate, was termed incurvated flexiated fibular osteotomy (IFFO) (Fig. 7). The average IFFO length was $23.9 \% \pm 6.7 \%$ relative to the fibular length. Postoperative and final long leg standing IFFO X-

Table 3. Comparison of Radiographic Parameters and Clinical Outcomes before and after Surgery in the Study Population

\begin{tabular}{lccc}
\hline \multicolumn{1}{c}{ Parameter } & Preoperative & Postoperative & p-value \\
\hline Femorotibial angle $\left(^{\circ}\right)$ & $180.7 \pm 3.8$ & $170.4 \pm 2.9$ & $<0.001$ \\
Mechanical axis $(\%)$ & $22.0 \pm 3.8$ & $60.2 \pm 10.5$ & $<0.001$ \\
Posterior tibial slope $\left(^{\circ}\right)$ & $11.5 \pm 3.1$ & $10.0 \pm 5.3$ & 0.094 \\
Insall-Salvati index & $0.9 \pm 0.1$ & $0.9 \pm 0.1$ & 0.647 \\
Caton-Deschamps index & $0.9 \pm 0.1$ & $0.9 \pm 0.1$ & 0.121 \\
Japanese Orthopedic & $72.8 \pm 14.9$ & $92.0 \pm 7.2$ & $<0.001$ \\
$\quad$ Association score & & & \\
Lysholm score & $66.6 \pm 11.0$ & $86.7 \pm 12.2$ & $<0.001$ \\
\hline
\end{tabular}

Values are presented as mean \pm standard deviation. rays are shown in Fig. 6. Two postoperative complications were observed around the tibial osteotomy in our cohort. One was an infected postoperative hematoma that needed surgical intervention involving debridement and plate removal. The other involved herniation of the anterior tibialis muscle that was revealed by mild ankle dorsal flexion power loss. However, this condition was resolved after primary repair of the fascia during implant removal (Fig. 8).

\section{Discussion}

Our present study is the first to report good to excellent clinical outcomes of a novel hybrid CWHTO procedure to treat medial $\mathrm{OA}$ of the knee. Corrections from $180.7^{\circ} \pm 3.8^{\circ}$ to $170.4^{\circ} \pm 2.9^{\circ}$ in the FTA and from $22.0 \% \pm 3.8 \%$ to $60.2 \% \pm 10.5 \%$ in the MA were achieved whilst maintaining the patella height and PTS with our new fibular osteotomy method.

Recently, the number of surgeons using OWHTO has increased because it is a comparatively simpler technique than $\mathrm{CWH}-$ $\mathrm{TO}^{3,5,8,12,23)}$. OWHTO is most effective during the early or middle stages of knee OA. However, it is not expected to have a beneficial impact if the knee $\mathrm{OA}$ is accompanied by a severe deformity or patellofemoral joint $\mathrm{OA}^{10)}$, partly because a huge bone gap is required to correct severe deformities. In such conditions, the soft exposed tissue around the knees creates a potentially dangerous
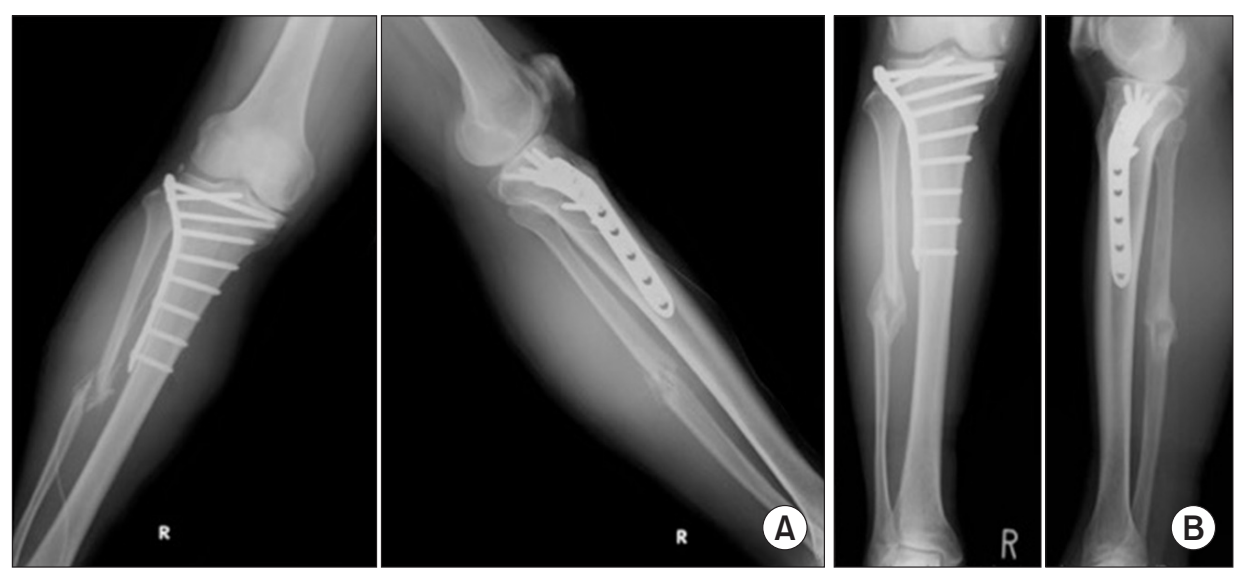

Fig. 7. Postoperative and final radiographs after the new fibula osteotomy technique. (A) X-ray immediately after incurvated flexiated fibular osteotomy. (B) Fibula united at final follow-up X-ray.
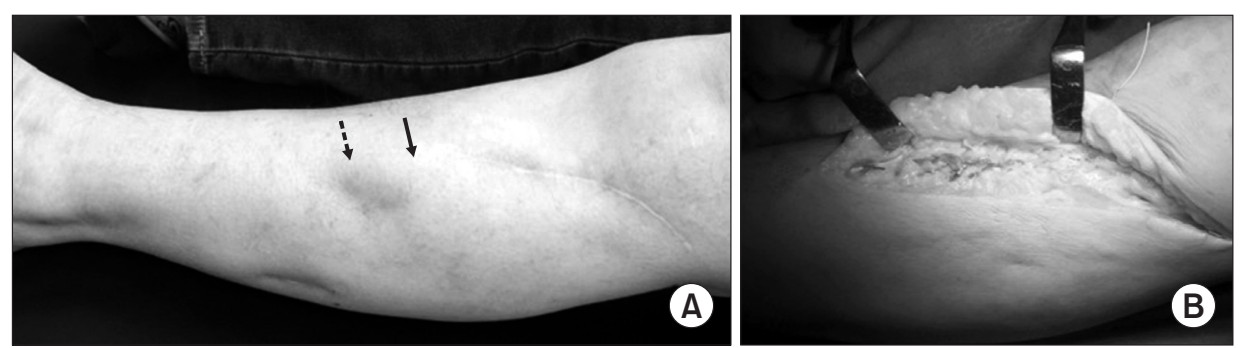

Fig. 8. A complication case of tibialis anterior muscle herniation. (A) Muscle bulging (solid arrow) and dimple (dotted arrow) due to muscle herniation were observed in the anterolateral tibia. (B) Muscle hernia was confirmed and the fascia was primarily repaired by running suture using a \#2 nonabsorbable thread. 
situation: an increased risk of infection or lateral hinge fracture, and delay in bone union. Furthermore, OWHTO is not recommended for knees with an associated patellofemoral joint OA because the decreased patellar height after this procedure might worsen the patellofemoral joint symptoms ${ }^{24)}$.

We agree with the proposition that the hybrid CWHTO procedure could be feasible in the treatment of medial compartment $\mathrm{OA}$ that requires extensive correction and is associated with patellofemoral OA, even if the standing FTA is more than $185^{\circ}$, the MA passes far from the medial margin of the tibial plateau, or patella baja is noted. The outcomes we observed in our current analyses support this proposition because the hybrid CWHTO procedure did not change the patellar height or posterior slope of the tibia based on the radiographic assessment of the patient knees in our present series. In fact, the radiographic grade of PF compartment in 27 of 29 knees (93.1\%) was unchanged except
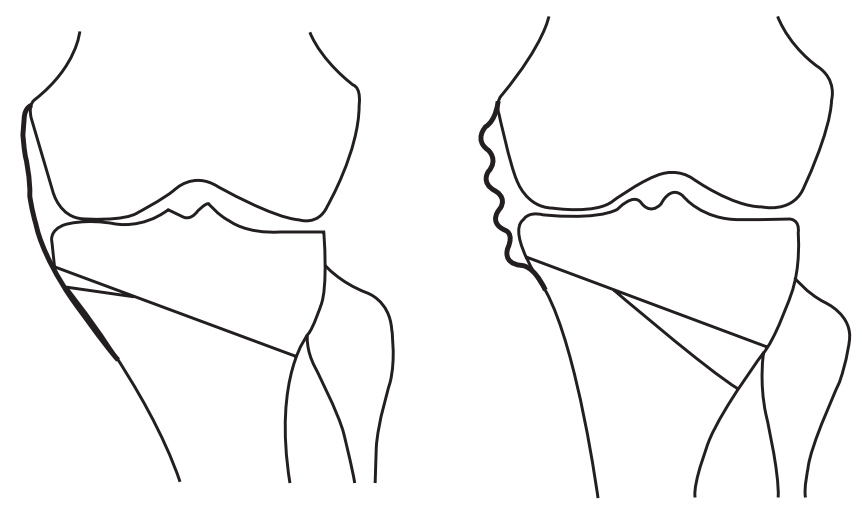

Fig. 9. Schematic drawing of re-tensioning of a slackened medial collateral ligament (MCL). The MCL was slack prior to hybrid closed-wedge high tibial valgus osteotomy due to cartilage loss. The hybrid procedure re-tensioned the MCL through creation of a medial gap opening. for 2 knees that underwent a simultaneous ACL reconstruction using a BTB graft, although none of the knees we analyzed had patellofemoral clinical symptoms. Notably, all reconstructions were made with an ipsilateral BTB graft without accelerated postoperative knee rehabilitation, which is a known risk factor for postoperative patellofemoral symptoms. This may be an important risk factor for the extension deficit and patellofemoral joint OA observed in this study and in previous studies that reported complications of bone patella-tendon-bone graft during ACL reconstruction on the patellofemoral joint as graft site morbidity ${ }^{25)}$. With regard to OWHTO, Stoffel et al. ${ }^{26)}$ have investigated patellofemoral pressure in their prior cadaveric study and reported that the pressure associated with OWHTO was significantly higher at $15^{\circ}, 30^{\circ}$, or $60^{\circ}$ flexion compared with CWHTO. Oh et al. ${ }^{20)}$ reported that the patellofemoral OA grade progressed or worsened in 15 of 42 knees (35.7\%) after OWHTO over a minimum follow-up of 5 years. Thus, hybrid CWHTO may be beneficial for treating medial OA patients with extensive deformities associated with patellofemoral OA or patella baja.

Preserving the medial soft tissues has the great advantage of enhancing the bone healing potential at the osteotomy site. On the other hand, we were concerned that the higher tension of the MCL after hybrid CWHTO would increase the pressure of the medial compartment and might not relieve the pain. Because pressure in the medial compartment increases during open-wedge osteotomy under an intact MCL, transection of the MCL is necessary to reduce this pressure ${ }^{27)}$. Takeuchi et al. ${ }^{10)}$ speculated that an increased tension of the superficial bundle of the slackened MCL due to some cartilage loss would improve the stability of the medial compartment joint and diminish knee pain. We agree with this hypothesis because the hybrid CWHTO procedure not only changes the tibial alignment but also stretches the slackened
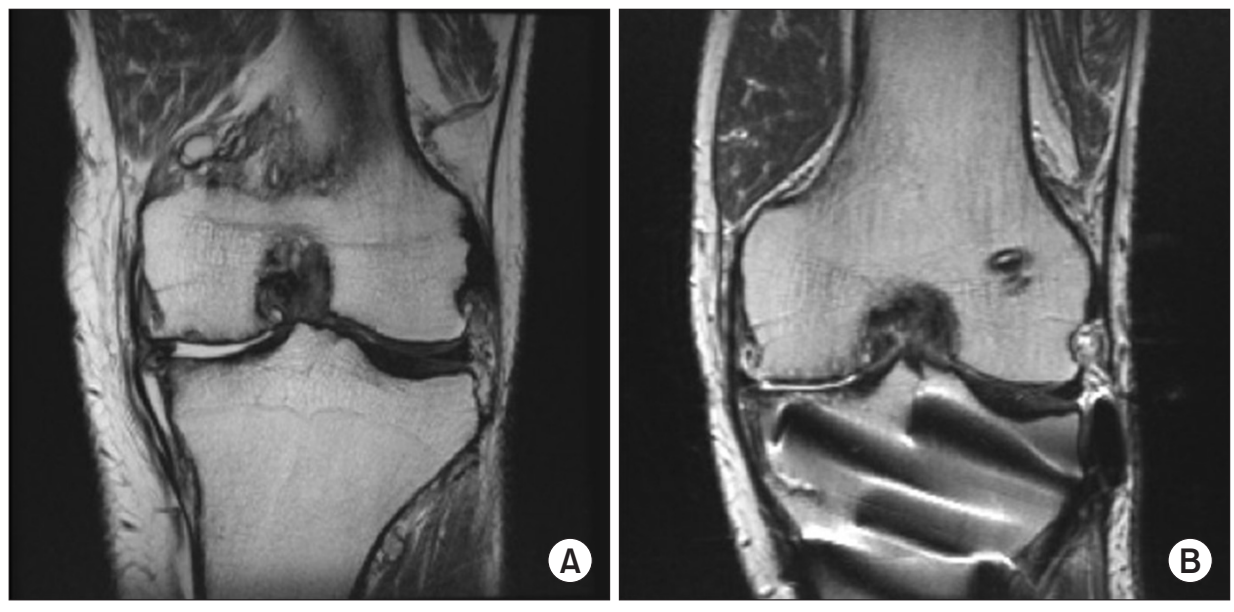

Fig. 10. Slackened medial collateral ligament (MCL) stretched over hybrid closed-wedge high tibial valgus osteotomy (CWHTO). (A) The MCL was slackened before osteotomy. (B) Stretched and re-tensioned MCL was observed after hybrid CWHTO. 
MCL to as far as the medial gap created at the osteotomy site (Fig. $9)^{28)}$. Fortunately, we could obtain evidence of the retension effect on the MCL which was revealed to have slackened prior to the hybrid CWHTO (Fig. 10A) but pulled at both ends and retensioned after the osteotomy (Fig. 10B). This effect on the MCL is specific evidence of the ability of the hybrid CWHTO to improve stability over the course of the procedure. Our present results further support this hypothesis because the clinical scores in all of our subject knees, including the pain score, showed significant improvement. Thus, given the effect of this hybrid procedure on deformity correction, we propose that it could potentially correct not only extra-articular deformities, such as tibia vara, but also mild intraarticular deformities due to cartilage loss, which is attributable to the appropriate level of retensioning of the slackened MCL.

We could not define a distinct surgical indication for hybrid CWHTO based on our own observations and experience. However, it has been established that an expertly performed osteotomy at a double level preserving the optimal axial alignment and horizontal joint line obliquity improves the long-term results with $100 \%$ survival rate and with no requirement for a TKA revision or instance of severe pain in any of the treated patients at 10 years after surgery ${ }^{29)}$. Thus, not only the MA but also an appropriate joint line orientation is one of the key elements to the success of this surgery. Nakayama et al. ${ }^{30)}$ and colleagues have previously investigated the stress induced by joint-line obliquity after large correction OWHTO in a three-dimensional finite element model analysis. These authors reported that a joint line obliquity of $5^{\circ}$ or more may induce detrimental stress at the articular cartilage, and they recommended consideration of a double level osteotomy as a viable surgical option in this situation. From the perspective of an optimal axial alignment, a hybrid CWHTO can cover and set the MA at the ideal point (around \%MA 60) even in cases of excessive varus alignment. However, with regard to joint line orientation, a horizontal joint line cannot be maintained after a large correction in a more extensive varus alignment. Moreover, leg length discrepancies have to be considered after any large correction.

Our present study had several limitations of note. First, it contained a relatively small sample size and involved relatively shortterm results (the maximum follow-up was 68.1 months). Nonetheless, our current findings represent the first clinical results of this new surgical procedure. Second, we did not include patientreported functional results, such as the Oxford knee score. Thus, further studies that assess a larger sample size with longer term results and patient-based outcomes are needed. Finally, although our results are related to the modified CWHTO surgical technique as the potentially most optimal osteotomy technique and concept, we did not perform a comparison of OWHTO, CWHTO, and hybrid CWHTO, because we have no data for conventional CWHTO. Therefore, we are unable at this stage to definitively conclude that the hybrid CWHTO is superior to all other procedures for treating medial knee OA.

\section{Conclusions}

Our present series of medial knee OA cases that underwent the hybrid CWHTO showed satisfactory outcomes at an average follow-up of 52.6 months. The postoperative PTS and patella height did not change in these cases compared with the preoperative assessment.

\section{Conflict of Interest}

No potential conflict of interest relevant to this article was reported.

\section{References}

1. Hui C, Salmon LJ, Kok A, Williams HA, Hockers N, van der Tempel WM, Chana R, Pinczewski LA. Long-term survival of high tibial osteotomy for medial compartment osteoarthritis of the knee. Am J Sports Med. 2011;39:64-70.

2. Schallberger A, Jacobi M, Wahl P, Maestretti G, Jakob RP. High tibial valgus osteotomy in unicompartmental medial osteoarthritis of the knee: a retrospective follow-up study over 13-21 years. Knee Surg Sports Traumatol Arthrosc. 2011;19:122-7.

3. Schroter S, Ateschrang A, Löwe W, Nakayama H, Stockle U, Ihle C. Early full weight-bearing versus 6-week partial weight-bearing after open wedge high tibial osteotomy leads to earlier improvement of the clinical results: a prospective, randomised evaluation. Knee Surg Sports Traumatol Arthrosc. 2017;25:325-32.

4. Takeuchi R, Ishikawa H, Kumagai K, Yamaguchi Y, Chiba N, Akamatsu Y, Saito T. Fractures around the lateral cortical hinge after a medial opening-wedge high tibial osteotomy: a new classification of lateral hinge fracture. Arthroscopy. 2012;28:85-94

5. Staubli AE, De Simoni C, Babst R, Lobenhoffer P. TomoFix: a new LCP-concept for open wedge osteotomy of the medial proximal tibia: early results in 92 cases. Injury. 2003;34 Suppl 
2:B55-62.

6. Efe T, Ahmed G, Heyse TJ, Boudriot U, Timmesfeld N, Fuchs-Winkelmann S, Ishaque B, Lakemeier S, Schofer MD. Closing-wedge high tibial osteotomy: survival and risk factor analysis at long-term follow up. BMC Musculoskelet Disord. 2011;12:46.

7. Bode G, von Heyden J, Pestka J, Schmal H, Salzmann G, Südkamp N, Niemeyer P. Prospective 5-year survival rate data following open-wedge valgus high tibial osteotomy. Knee Surg Sports Traumatol Arthrosc. 2015;23:1949-55.

8. Floerkemeier S, Staubli AE, Schroeter S, Goldhahn S, Lobenhoffer P. Outcome after high tibial open-wedge osteotomy: a retrospective evaluation of 533 patients. Knee Surg Sports Traumatol Arthrosc. 2013;21:170-80.

9. Giuseffi SA, Replogle WH, Shelton WR. Opening-wedge high tibial osteotomy: review of 100 consecutive cases. Arthroscopy. 2015;31:2128-37.

10. Takeuchi R, Ishikawa H, Miyasaka Y, Sasaki Y, Kuniya T, Tsukahara S. A novel closed-wedge high tibial osteotomy procedure to treat osteoarthritis of the knee: hybrid technique and rehabilitation measures. Arthrosc Tech. 2014;3: e431-7.

11. Pape D, Lorbach O, Schmitz C, Busch LC, Van Giffen N, Seil R, Kohn DM. Effect of a biplanar osteotomy on primary stability following high tibial osteotomy: a biomechanical cadaver study. Knee Surg Sports Traumatol Arthrosc. 2010; 18:204-11.

12. Lobenhoffer P, Agneskirchner JD. Improvements in surgical technique of valgus high tibial osteotomy. Knee Surg Sports Traumatol Arthrosc. 2003;11:132-8.

13. Amis AA. Biomechanics of high tibial osteotomy. Knee Surg Sports Traumatol Arthrosc. 2013;21:197-205.

14. Bin SI, Kim HJ, Ahn HS, Rim DS, Lee DH. Changes in patellar height after opening wedge and closing wedge high tibial osteotomy: a meta-analysis. Arthroscopy. 2016;32:2393-400.

15. Shino K, Nakata K, Nakamura N, Toritsuka Y, Horibe S, Nakagawa S, Suzuki T. Rectangular tunnel double-bundle anterior cruciate ligament reconstruction with bone-patellar tendon-bone graft to mimic natural fiber arrangement. Arthroscopy. 2008;24:1178-83.

16. Outerbridge RE. The etiology of chondromalacia patellae. J Bone Joint Surg Br. 1961;43:752-7.

17. Miniaci A, Ballmer FT, Ballmer PM, Jakob RP. Proximal tibial osteotomy: a new fixation device. Clin Orthop Relat Res. 1989;(246):250-9.

18. Shino K, Mitsuoka T, Horibe S, Hamada M, Nakata K,
Nakamura N. The gravity sag view: a simple radiographic technique to show posterior laxity of the knee. Arthroscopy. 2000;16:670-2.

19. Majima T, Yasuda K, Aoki Y, Minami A. Impact of patellofemoral osteoarthritis on long-term outcome of high tibial osteotomy and effects of ventralization of tibial tubercle. J Orthop Sci. 2008;13:192-7.

20. Oh KJ, Kim YC, Lee JS, Chang YS, Shetty GM, Nha KW. Open-wedge high tibial osteotomy versus unicompartmental knee arthroplasty: no difference in progression of patellofemoral joint arthritis. Knee Surg Sports Traumatol Arthrosc. 2017;25:767-72.

21. Lysholm J, Gillquist J. Evaluation of knee ligament surgery results with special emphasis on use of a scoring scale. Am J Sports Med. 1982;10:150-4.

22. Okuda M, Omokawa S, Okahashi K, Akahane M, Tanaka Y. Validity and reliability of the Japanese Orthopaedic Association score for osteoarthritic knees. J Orthop Sci. 2012;17:7506.

23. Takeuchi R, Ishikawa $H$, Aratake M, Bito H, Saito I, Kumagai K, Akamatsu Y, Saito T. Medial opening wedge high tibial osteotomy with early full weight bearing. Arthroscopy. 2009;25:46-53.

24. El-Azab H, Glabgly P, Paul J, Imhoff AB, Hinterwimmer S. Patellar height and posterior tibial slope after open- and closed-wedge high tibial osteotomy: a radiological study on 100 patients. Am J Sports Med. 2010;38:323-9.

25. Shino K, Nakagawa S, Inoue M, Horibe S, Yoneda M. Deterioration of patellofemoral articular surfaces after anterior cruciate ligament reconstruction. Am J Sports Med. 1993; 21:206-11.

26. Stoffel K, Willers C, Korshid O, Kuster M. Patellofemoral contact pressure following high tibial osteotomy: a cadaveric study. Knee Surg Sports Traumatol Arthrosc. 2007;15:1094100.

27. Agneskirchner JD, Hurschler C, Wrann CD, Lobenhoffer P. The effects of valgus medial opening wedge high tibial osteotomy on articular cartilage pressure of the knee: a biomechanical study. Arthroscopy. 2007;23:852-61.

28. Goodfellow J. Unicompartmental arthroplasty with the Oxford knee. Oxford: Oxford University Press; 2006. p71.

29. Babis GC, An KN, Chao EY, Larson DR, Rand JA, Sim FH. Upper tibia osteotomy: long term results: realignment analysis using OASIS computer software. J Orthop Sci. 2008;13: 328-34.

30. Nakayama H, Schroter S, Yamamoto C, Iseki T, Kanto R, Ku- 
302 Saito et al. Short-Term Results of Hybrid Closed-Wedge High Tibial Osteotomy

rosaka K, Kambara S, Yoshiya S, Higa M. Large correction in opening wedge high tibial osteotomy with resultant jointline obliquity induces excessive shear stress on the articular cartilage. Knee Surg Sports Traumatol Arthrosc. 2018;26: 1873-8. 